ARTICLE

https://doi.org/10.1038/s41467-021-24400-5

\title{
Electrochemical synthesis of urea on MBenes
}

\author{
Xiaorong Zhu (1) ${ }^{1}$ Xiaocheng Zhou', Yu Jing ${ }^{2} \&$ Yafei Li ${ }^{1 凶}$
}

Urea is an important raw material in the chemical industry and is widely used as a nitrogen source in chemical fertilizers. The current industrial urea synthesis not only requires harsh reaction conditions, but also consumes most of the $\mathrm{NH}_{3}$ obtained through artificial synthesis. The conversion of $\mathrm{N}_{2}$ and $\mathrm{CO}_{2}$ into urea through electrochemical reactions under ambient conditions represents a novel green urea synthesis method. However, the large-scale promotion of this method is limited by the lack of suitable electrocatalysts. Here, by means of density functional theory computations, we systematically study the catalytic activity of three experimentally available two-dimensional metal borides (MBenes), $\mathrm{Mo}_{2} \mathrm{~B}_{2}, \mathrm{Ti}_{2} \mathrm{~B}_{2}$, and $\mathrm{Cr}_{2} \mathrm{~B}_{2}$ toward simultaneous electrocatalytic coupling of $\mathrm{N}_{2}$ and $\mathrm{CO}_{2}$ to produce urea under ambient conditions. According to our results, these three MBenes not only have superior intrinsic basal activity for urea formation, with limiting potentials ranging from -0.49 to $-0.65 \mathrm{eV}$, but also can significantly suppress the competitive reaction of $\mathrm{N}_{2}$ reduction to $\mathrm{NH}_{3}$. In particular, 2D Mo $\mathrm{B}_{2}$ and $\mathrm{Cr}_{2} \mathrm{~B}_{2}$ possess superior capacity to suppress surface oxidation and self-corrosion under electrochemical reaction conditions, rendering them relatively promising electrocatalysts for urea production. Our work paves the way for the electrochemical synthesis of urea.

\footnotetext{
${ }^{1}$ Jiangsu Collaborative Innovation Centre of Biomedical Functional Materials, School of Chemistry and Materials Science, Nanjing Normal University, Nanjing, China. ${ }^{2}$ College of Chemical Engineering, Nanjing Forestry University, Nanjing, China. ${ }^{凶}$ email: liyafei@njnu.edu.cn
} 
U rea, also known as carbamide $\left(\mathrm{CO}\left(\mathrm{NH}_{2}\right)_{2}\right)$, was the first organic compound produced from inorganic raw materials. Because its nitrogen content is high (46\%) and is readily converted to ammonia $\left(\mathrm{NH}_{3}\right)$ in the soil, urea is now the most commonly used nitrogen fertilizer in the world, and more urea is manufactured by mass than any other organic chemical ${ }^{1}$. While over $90 \%$ of produced urea is used as fertilizer, it also has important applications in other fields. For example, urea is a raw material for the manufacture of urea-formaldehyde and ureamelamine-formaldehyde resins ${ }^{2}$. Urea-containing creams are used as topical dermatological products to promote skin hydration $^{3}$, and large amounts of urea are used for the synthesis of barbiturates ${ }^{4}$. Since $\mathrm{NH}_{3}$ produced by the hydrolysis of urea can react with nitrogen oxides $\left(\mathrm{NO}_{\mathrm{x}}\right)$ to produce nitrogen, an increasingly important application of urea is to reduce $\mathrm{NO}_{\mathrm{x}}$ impurities in exhaust gases from diesel and lean-burn natural gas engines $^{5}$. Therefore, maintaining a sustainable and efficient urea industry is of great importance for the development of human society.

At present, the production of urea in the industry is accomplished mainly through the reaction of $\mathrm{NH}_{3}$ and $\mathrm{CO}_{2}$ under high temperature and high pressure. However, this method is not only relatively energy-consuming but also relies on some complex types of equipment and multicycle processes to improve the conversion efficiency ${ }^{6,7}$. Remarkably, urea production consumes $\sim 80 \%$ of the global $\mathrm{NH}_{3}$, which is mainly derived from the artificial nitrogen reduction reaction $(\mathrm{NRR})^{8}$. Nevertheless, $\mathrm{N}_{2}$ is a stable molecule, and substantial input energy is required for dissociation of the strong $\mathrm{N} \equiv \mathrm{N}$ triple bond, making $\mathrm{N}_{2}$ reduction thermodynamically and kinetically very difficult ${ }^{9}$. The industrial NRR is still dominated by the traditional Haber-Bosch process, which converts $\mathrm{N}_{2}$ and $\mathrm{H}_{2}$ into $\mathrm{NH}_{3}$ with the assistance of ironbased catalysts under harsh conditions ${ }^{10,11}$. However, the very large energy consumption and the large amount of the greenhouse gas $\mathrm{CO}_{2}$ emitted by the Haber-Bosch process have aggravated the energy and environmental problems. Therefore, people have been striving for green ammonia synthetic techniques that can be carried out under mild conditions ${ }^{12-14}$.

Compared to the Haber-Bosch process, the production of $\mathrm{NH}_{3}$ through the electrochemical NRR represents a more efficient and green strategy as it can utilize electricity generated from renewable energy sources and protons directly from water ${ }^{15-20}$. However, separating and purifying $\mathrm{NH}_{3}$ from an aqueous electrolyte is very difficult, which is detrimental to its further application. Furthermore, most current studies mainly focus on $\mathrm{N}_{2}$ electrochemical reduction to $\mathrm{NH}_{3}$, while further processing of the product is rarely considered. Recently, Jouny et al. ${ }^{21}$ realized $\mathrm{C}-\mathrm{N}$ coupling and the production of acetamides with a high rate and selectivity by using $\mathrm{NH}_{3}$ as a nitrogen source. More interestingly, Comer et al. ${ }^{22}$ demonstrated that during photocatalytic fixation on the surface of $\mathrm{TiO}_{2}, \mathrm{~N}_{2}$ can have a strong interaction with carbon substitution sites that present as surface-bound radicals, implying the feasibility of direct formation of $\mathrm{C}-\mathrm{N}$ bonds from the coupling of $\mathrm{N}_{2}$ and carbon-based reagents. Inspired by these pioneering works, Chen et al. ${ }^{23}$ recently successfully coupled $\mathrm{N}_{2}$ and $\mathrm{CO}_{2}$ in $\mathrm{H}_{2} \mathrm{O}$ to produce urea using an electrocatalyst consisting of $\mathrm{Pd}-\mathrm{Cu}$ alloy nanoparticles on $\mathrm{TiO}_{2}$ nanosheets, which opens a new avenue for urea production under mild conditions. However, in addition to the high price, precious metal-based alloy catalysts usually suffer from ambiguous active sites and easy corrosion. Therefore, for the development of emerging electrochemical urea synthesis, inexpensive and efficient electrocatalysts that can fix $\mathrm{N}_{2}$ and $\mathrm{CO}_{2}$ together are highly desirable.

In the past decade, the application of two-dimensional (2D) materials in the field of electrocatalysis have received much attention due to their large specific surface area and more exposed active sites ${ }^{24,25}$. For example, Li et al. ${ }^{26}$ theoretically demonstrated that 2D transition metal carbides, namely MXenes, are capable of catalyzing the conversion of $\mathrm{CO}_{2}$ into hydrocarbons. However, the surface metal atoms of MXenes are easily passivated by some functional species (e.g. $\mathrm{OH}, \mathrm{F}, \mathrm{O}$ ), thereby degrading the catalytic activity ${ }^{27-29}$. Recently, several 2D transition metal borides (MBenes), which are boron analogs of MXenes, have also been realized experimentally ${ }^{30-33}$. In contrast to MXenes, MBenes can be stabilized without the presence of surface passivation groups. Therefore, MBenes provide an ideal platform for the exploration of the catalytic behavior of boron-containing surfaces ${ }^{34-36}$. Some recent theoretical studies have demonstrated that several types of MBenes could present good activity and selectivity for the $\mathrm{NRR}^{37,38}$. Motivated by the regulated layered configuration and excellent electrical conductivity of MBenes, we wondered whether the direct coupling of $\mathrm{N}_{2}$ and $\mathrm{CO}_{2}$ to produce urea could be realized on some specific MBenes.

In this work, by means of density functional theory (DFT) computations, we show that the electrochemical synthesis of urea on the basal planes of three experimentally realized MBenes, including $2 \mathrm{D} \mathrm{Mo}_{2} \mathrm{~B}_{2}, \mathrm{Ti}_{2} \mathrm{~B}_{2}$, and $\mathrm{Cr}_{2} \mathrm{~B}_{2}$ is thermodynamically and kinetically favorable. Remarkably, the competitive NRR can be significantly overwhelmed on these three MBenes, suggesting good selectivity. Especially, the surface oxidation/degradation problem can be avoided on the surfaces of $\mathrm{Mo}_{2} \mathrm{~B}_{2}$ and $\mathrm{Cr}_{2} \mathrm{~B}_{2}$, endowing these two MBenes with intrinsic activity, selectivity, and a large reaction region toward electrochemical urea synthesis.

\section{Results}

Structural properties and stability of MBenes. Figure 1 shows the schematic structure of a $2 \mathrm{D} \mathrm{M}_{2} \mathrm{~B}_{2}$-type MBene. Unlike many ordinary $2 \mathrm{D}$ materials (e.g., graphene, $\mathrm{MoS}_{2}$, MXenes) that possess a hexagonal lattice, our three studied 2D MBenes all present a rectangular lattice with the space group Pmma (No. 51), resulting in an in-plane structural anisotropy. The unit cell of a $2 \mathrm{D} \mathrm{M}_{2} \mathrm{~B}_{2}$ type MBene consists of two metal atoms and two $\mathrm{B}$ atoms, and each metal atom or B atom is connected to six neighboring atoms,

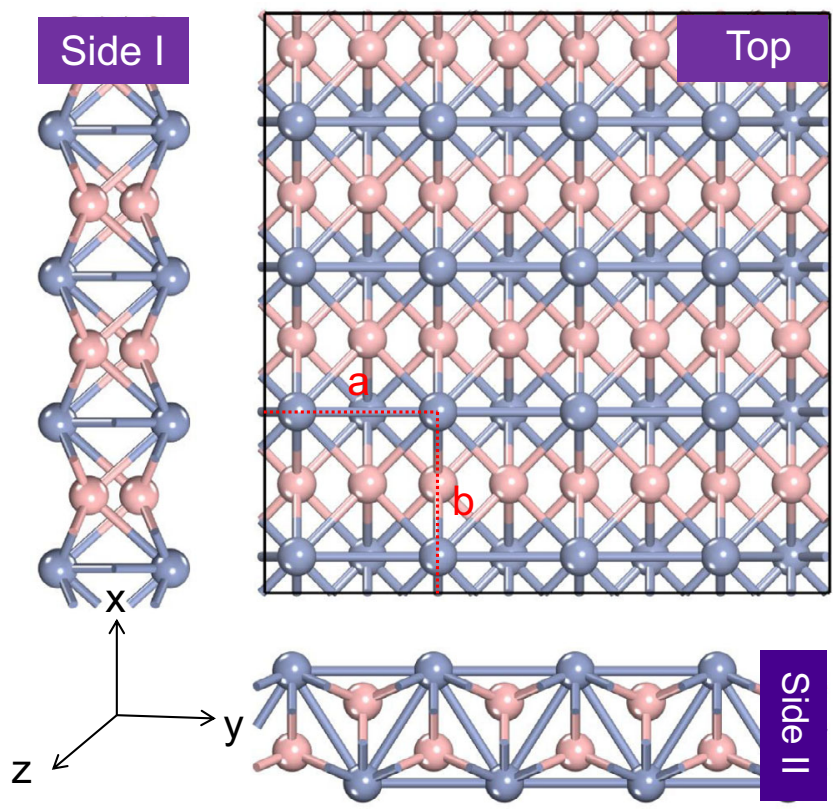

Fig. 1 Geometrics of MBenes. Top and side views of the schematic structure of a $\mathrm{M}_{2} \mathrm{~B}_{2}$-type MBene. The red dashed lines denote a unit cell. The pink and blue balls represent boron and transition metal atoms, respectively. 


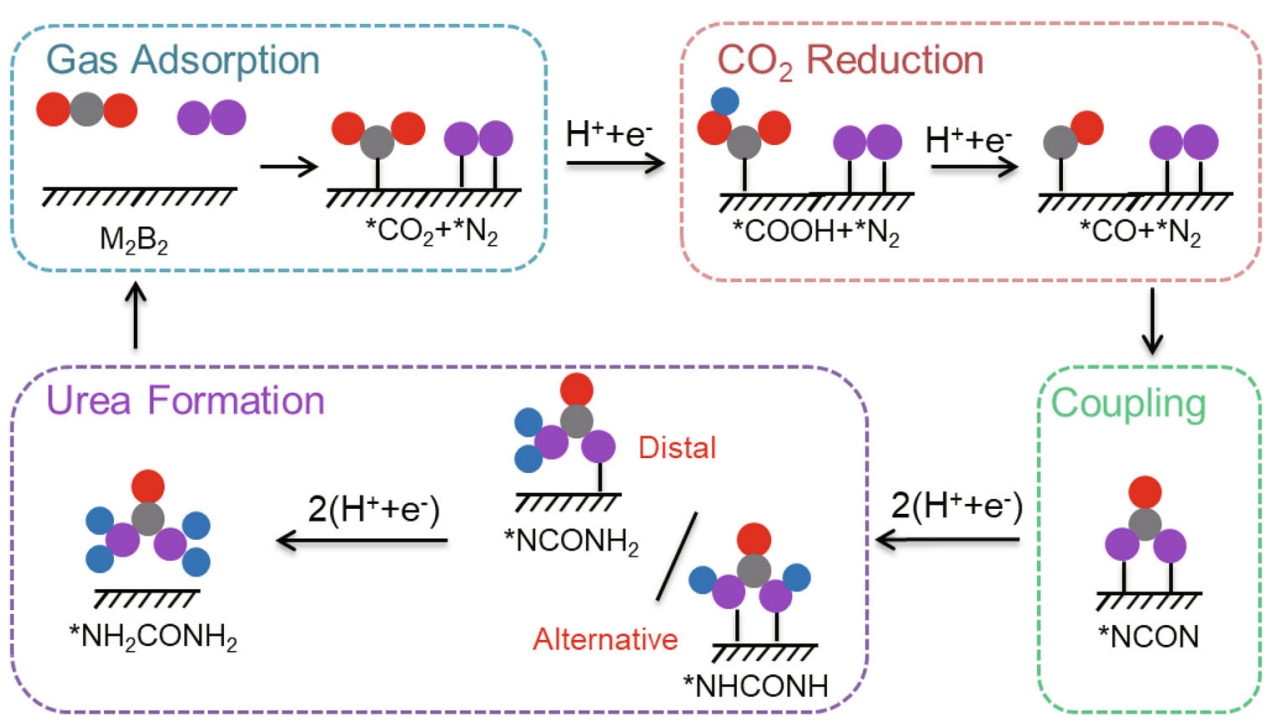

Fig. 2 Reaction mechanism. Schematic diagram of the mechanism of urea production through the electrochemical coupling of $\mathrm{N}_{2}$ and $\mathrm{CO}_{2}$. The gray, red, pink, and blue balls represent $\mathrm{C}, \mathrm{O}, \mathrm{N}$, and $\mathrm{H}$ atoms, respectively.

forming a buckled bilayer structure with metal atoms on the uppermost surface. Compared to MXenes, in which the surface metal atoms bind to three carbon or nitrogen atoms, the higher coordination number of surface metal atoms in $\mathrm{M}_{2} \mathrm{~B}_{2}$-type MBenes endow them with distinct stability and properties. The optimized lattice parameters and representative bond lengths of $\mathrm{Mo}_{2} \mathrm{~B}_{2}, \mathrm{Ti}_{2} \mathrm{~B}_{2}$, and $\mathrm{Cr}_{2} \mathrm{~B}_{2}$ MBenes are summarized in Supplementary Table 1 . Moreover, the electronic band structure computations demonstrated that these three MBenes are all metallic (Supplementary Fig. 1), which is beneficial for their electrocatalytic activity. The above results achieved good agreement with previous studies 39,40 .

Good stability is a prerequisite for the wide utilization of a catalyst. We thus first assessed the stability of $2 \mathrm{D} \mathrm{Mo}_{2} \mathrm{~B}_{2}, \mathrm{Ti}_{2} \mathrm{~B}_{2}$, and $\mathrm{Cr}_{2} \mathrm{~B}_{2}$ MBenes before revealing their catalytic activity toward urea formation. As shown in Supplementary Fig. 2, no imaginary modes are found in the phonon spectra of the three MBenes, which is indicative of good kinetic stability. The elastic constants of the three monolayers presented in Supplementary Table 2 all meet the criteria for a rectangular $2 \mathrm{D}$ structure $\left(\mathrm{C}_{11} \mathrm{C}_{22}-\mathrm{C}_{12}{ }^{2}>\right.$ $\left.0, \mathrm{C}_{66}>0\right)^{39}$, indicating that they are mechanically stable. Moreover, we also performed first-principles molecular dynamics (FPMD) simulations to examine the thermal stability. According to the diagrams presented in Supplementary Fig. 3, no obvious structural deformation can be observed in the structures of the three MBenes after 10 ps FPMD simulations at $300 \mathrm{~K}$, suggesting good thermal stability.

Mechanism of electrochemical urea synthesis. To date, the reaction mechanism for electrochemical $\mathrm{N}_{2}$ and $\mathrm{CO}_{2}$ coupling to produce urea has only been proposed by Chen et al. ${ }^{23}$. As shown in Fig. 2, the entire reaction can be divided into four stages, namely, the adsorption of $\mathrm{N}_{2}$ and $\mathrm{CO}_{2}$, the reduction of ${ }^{*} \mathrm{CO}_{2}$ to ${ }^{*} \mathrm{CO}$, the coupling of ${ }^{*} \mathrm{~N}_{2}$ and ${ }^{*} \mathrm{CO}$ into ${ }^{*} \mathrm{NCON}$, and the hydrogenation of $* \mathrm{NCON}$ to urea. Specifically, effective adsorption of $\mathrm{N}_{2}$ and $\mathrm{CO}_{2}$ on the surface of the catalyst is the primary condition for electrochemical urea production. Moreover, the adsorbed $\mathrm{N}_{2}$ should not be reduced to $\mathrm{NH}_{3}$, or at least the limiting potential for urea formation $\left(U_{L}^{\text {urea }}\right)$ should be lower than that of $\mathrm{NH}_{3}$ formation $\left(U_{L}^{\mathrm{NH}_{3}}\right)$ to guarantee a high selectivity. Once $\mathrm{N}_{2}$ is adsorbed, the coadsorbed $\mathrm{CO}_{2}$ should be effectively

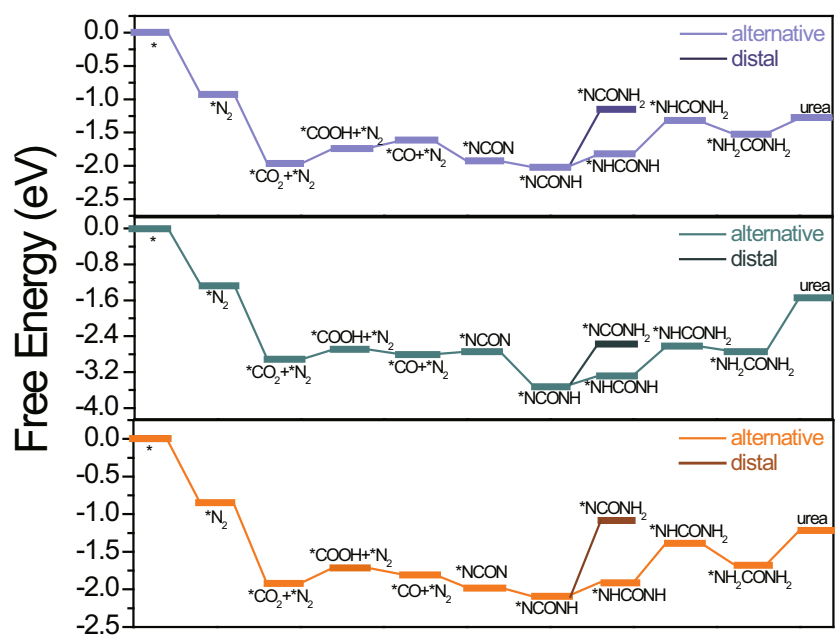

Reaction Coordinate

Fig. 3 Catalytic activity of MBenes. Free energy profiles of electrochemical urea production on $\mathrm{Mo}_{2} \mathrm{~B}_{2}, \mathrm{Ti}_{2} \mathrm{~B}_{2}$, and $\mathrm{Cr}_{2} \mathrm{~B}_{2}$.

and selectively reduced to ${ }^{*} \mathrm{CO}$. Then, instead of being released or further reduced, the generated ${ }^{*} \mathrm{CO}$ should move to the top of adsorbed ${ }^{*} \mathrm{~N}_{2}$ to form the tower-like key intermediate ${ }^{*} \mathrm{NCON}$. Finally, the formed ${ }^{*} \mathrm{NCON}$ species could be further reduced to urea via four proton-coupled electron transfer (PCET) steps following either the distal or the alternative pathway. This mechanism has been well applied to explain the catalytic activity of the Pd-Cu catalyst. For the investigation of urea formation over the three $\mathrm{M}_{2} \mathrm{~B}_{2}$-type MBenes, we followed the above-discussed reaction mechanism. The free-energies of reaction intermediates (Supplementary Table 3 ) and the reaction free-energies $(\Delta G)$ of elementary steps for urea production on $2 \mathrm{D} \mathrm{Mo}_{2} \mathrm{~B}_{2}, \mathrm{Ti}_{2} \mathrm{~B}_{2}$, and $\mathrm{Cr}_{2} \mathrm{~B}_{2}$ were then computed. The optimized atomic configurations of various intermediates along the reaction pathway are displayed in Supplementary Fig. 4, and the corresponding free energy profiles are summarized in Fig. 3.

Electrochemical reactivity of MBenes toward urea production. We first investigated the adsorption of $\mathrm{N}_{2}$ and $\mathrm{CO}_{2}$ on the 
surfaces of the three MBenes. For the adsorption of $\mathrm{N}_{2}$, both sideon and end-on configurations were considered. According to our computations, the side-on adsorption of $\mathrm{N}_{2}$ on the bridge site of two metal atoms is preferred for all three MBenes (Supplementary Fig. 4). This is because the back-donation of electrons from the $d$-orbitals of metal atoms to the $\pi^{*}$ orbitals of $\mathrm{N}_{2}$ could be facilitated by side-on adsorption. The adsorption energies of $\mathrm{N}_{2}$ $\left(\triangle E_{\mathrm{N}_{2}}\right)$ on $2 \mathrm{D} \mathrm{Mo}_{2} \mathrm{~B}_{2}, \mathrm{Ti}_{2} \mathrm{~B}_{2}$, and $\mathrm{Cr}_{2} \mathrm{~B}_{2}$ are $-0.93,-1.27$, and $-0.85 \mathrm{eV}$, respectively. Due to the strong interaction between $\mathrm{N}_{2}$ and the three MBenes, the bond length of $\mathrm{N}_{2}$ is enlarged from $1.10 \AA$ to $1.21,1.22$, and $1.20 \AA$ on $2 \mathrm{D} \mathrm{Mo}_{2} \mathrm{~B}_{2}, \mathrm{Ti}_{2} \mathrm{~B}_{2}$, and $\mathrm{Cr}_{2} \mathrm{~B}_{2}$, respectively. Once $\mathrm{N}_{2}$ is adsorbed, the accommodation of a $\mathrm{CO}_{2}$ molecule on its neighboring bridge site is also feasible, with adsorption energies $\left(\triangle E_{\mathrm{CO}_{2}}\right)$ of $-1.04,-1.65$, and $-1.07 \mathrm{eV}$ for 2D $\mathrm{Mo}_{2} \mathrm{~B}_{2}, \mathrm{Ti}_{2} \mathrm{~B}_{2}$, and $\mathrm{Cr}_{2} \mathrm{~B}_{2}$, respectively. Remarkably, the adsorbed $\mathrm{CO}_{2}$ is pronouncedly bent due to the formation of chemical bonds between $\mathrm{C}$ and metal atoms. Overall, the above results vividly reveal that both $\mathrm{N}_{2}$ and $\mathrm{CO}_{2}$ can be strongly adsorbed and effectively activated on the surface of our three chosen MBenes.

After disclosing the adsorption behavior of $\mathrm{N}_{2}$ and $\mathrm{CO}_{2}$, we next assessed the feasibility of ${ }^{*} \mathrm{CO}_{2}$ reduction to ${ }^{*} \mathrm{CO}$ on the surface of the three MBenes in the presence of coadsorbed $\mathrm{N}_{2}$. Generally, the electroreduction of $* \mathrm{CO}_{2}$ to $* \mathrm{CO}$ initiates with the hydrogenation of one $\mathrm{O}$ atom through a PCET step, resulting in the formation of a ${ }^{*} \mathrm{COOH}$ species. According to our computations, this step is endothermic with a $\Delta G$ of $0.24,0.22$, and 0.21 $\mathrm{eV}$ for $2 \mathrm{D} \mathrm{Mo}_{2} \mathrm{~B}_{2}, \mathrm{Ti}_{2} \mathrm{~B}_{2}$, and $\mathrm{Cr}_{2} \mathrm{~B}_{2}$, respectively. Upon the second PCET, the ${ }^{*} \mathrm{COOH}$ species can be transformed to a ${ }^{*} \mathrm{CO}$ species by releasing a $\mathrm{H}_{2} \mathrm{O}$ molecule, which is endothermic by $0.10 \mathrm{eV}$ for $2 \mathrm{D} \mathrm{Mo}_{2} \mathrm{~B}_{2}$ but exothermic by -0.11 and $-0.08 \mathrm{eV}$ for $2 \mathrm{D} \mathrm{Ti}_{2} \mathrm{~B}_{2}$ and $\mathrm{Cr}_{2} \mathrm{~B}_{2}$, respectively. Therefore, the key intermediate * CO can be feasibly generated on the surfaces of the three MBenes. The adsorption energies of ${ }^{*} \mathrm{CO}$ species on $2 \mathrm{D} \mathrm{Mo} \mathrm{B}_{2}$, $\mathrm{Ti}_{2} \mathrm{~B}_{2}$, and $\mathrm{Cr}_{2} \mathrm{~B}_{2}$ are $-0.69,-1.53$, and $-0.94 \mathrm{eV}$, respectively, which are all higher than that of Pd-Cu catalyst $(-0.62 \mathrm{eV})^{23}$. Due to the low CO yield of Pd-Cu catalyst, we can expect that the *CO desorption can be significantly suppressed for three 2D MBenes, which could facilitate the following coupling process.

As revealed by Chen et al. ${ }^{23}$ the most important intermediate for urea formation is the tower-like ${ }^{*} \mathrm{NCON}$ species, which can be directly produced from the coupling of $* \mathrm{CO}$ and ${ }^{*} \mathrm{~N}_{2}$. We thus explored the feasibility of forming the ${ }^{*} \mathrm{NCON}$ species on the surfaces of the three MBenes using the CI-NEB method. As shown in Supplementary Fig. 5, the coupling of ${ }^{*} \mathrm{~N}_{2}$ and ${ }^{*} \mathrm{CO}$ into $* \mathrm{NCON}$ is exothermic by -0.30 and $-0.18 \mathrm{eV}$ on $2 \mathrm{D} \mathrm{Mo} \mathrm{B}_{2}$ and $\mathrm{Cr}_{2} \mathrm{~B}_{2}$, respectively, indicating that the formation of the *NCON species on these two MBenes is energetically favorable. In contrast, due to the relatively strong binding strength of the ${ }^{*} \mathrm{CO}$ species, the formation of ${ }^{*} \mathrm{NCON}$ on $\mathrm{Ti}_{2} \mathrm{~B}_{2}$ is slightly endothermic by $0.06 \mathrm{eV}$. Remarkably, the kinetic barriers for the formation of ${ }^{*} \mathrm{NCON}$ on $2 \mathrm{D} \mathrm{Mo}_{2} \mathrm{~B}_{2}, \mathrm{Ti}_{2} \mathrm{~B}_{2}$, and $\mathrm{Cr}_{2} \mathrm{~B}_{2}$ are 0.58 , 0.81 , and $0.71 \mathrm{eV}$ respectively, which are comparable to or even lower than that of the $\mathrm{Pd}-\mathrm{Cu}$ catalyst $(0.79 \mathrm{eV})^{23}$, indicating that the coupling of ${ }^{*} \mathrm{~N}_{2}$ and ${ }^{*} \mathrm{CO}$ on these three MBenes is kinetically feasible.

Once the ${ }^{*} \mathrm{NCON}$ species is formed, the formation of urea becomes very straightforward, although there exist different sequences of hydrogenation on the two $\mathrm{N}$ atoms. Remarkably, the hydrogenation of ${ }^{*} \mathrm{NCON}$ to ${ }^{*} \mathrm{NCONH}$ via a PCET is exothermic for all three MBenes with a $\Delta G$ of $-0.09,-0.76$, and $-0.12 \mathrm{eV}$ for $\mathrm{Mo}_{2} \mathrm{~B}_{2}, \mathrm{Ti}_{2} \mathrm{~B}_{2}$, and $\mathrm{Cr}_{2} \mathrm{~B}_{2}$, respectively. Notably, we also considered the hydrogenation of the $\mathrm{O}$ atom but found that it is relatively endothermic. When the second $\mathrm{H}$ is added to * NCONH, two possible reaction pathways may occur. One is the distal pathway in which the second $\mathrm{H}$ is added to the hydrogenated $\mathrm{N}$ atom in the last step to form the ${ }^{*} \mathrm{NCONH}_{2}$ species, and the other is the alternative path in which the second $\mathrm{H}$ is added to the bare $\mathrm{N}$ atom to form ${ }^{*} \mathrm{NHCONH}$ species. According to our computations, the formation of the distal product ${ }^{*} \mathrm{NCONH}_{2}$ is endothermic by as high as $0.88,0.93$, and $1.02 \mathrm{eV}$ for $2 \mathrm{D} \mathrm{Mo}_{2} \mathrm{~B}_{2}, \mathrm{Ti}_{2} \mathrm{~B}_{2}$ and $\mathrm{Cr}_{2} \mathrm{~B}_{2}$, respectively. In contrast, the formation of the alternative product ${ }^{*} \mathrm{NHCONH}$ is $0.68,0.70$, and $0.83 \mathrm{eV}$ lower in energy than that of the distal product for $2 \mathrm{D}$ $\mathrm{Mo}_{2} \mathrm{~B}_{2}, \mathrm{Ti}_{2} \mathrm{~B}_{2}$, and $\mathrm{Cr}_{2} \mathrm{~B}_{2}$, respectively. As a comparison, the distal product is preferred by the $\mathrm{Pd}-\mathrm{Cu}$ catalyst ${ }^{23}$. Additionally, we noted that at this stage, the chemical bonding between metal atoms and $\mathrm{N}$ atoms is still robust, while the $\mathrm{N}-\mathrm{N}$ length in * NHCONH is enlarged to 2.35, 2.34, and $2.33 \AA$ for $2 \mathrm{D} \mathrm{Mo}_{2} \mathrm{~B}_{2}$, $\mathrm{Ti}_{2} \mathrm{~B}_{2}$, and $\mathrm{Cr}_{2} \mathrm{~B}_{2}$, respectively. Due to the breaking of one $\mathrm{M}-\mathrm{N}$ bond, the third PCET step to form ${ }^{*} \mathrm{NH}_{2} \mathrm{CONH}$ is considerably endothermic, with a $\Delta G$ of $0.49,0.65$, and $0.52 \mathrm{eV}$ for $2 \mathrm{D} \mathrm{Mo}_{2} \mathrm{~B}_{2}$, $\mathrm{Ti}_{2} \mathrm{~B}_{2}$, and $\mathrm{Cr}_{2} \mathrm{~B}_{2}$, respectively. Since the fourth PCET step to form ${ }^{*} \mathrm{NH}_{2} \mathrm{CONH}_{2}$ is exothermic for all three $\mathrm{M}_{2} \mathrm{~B}_{2}$ monolayers, the formation of the ${ }^{*} \mathrm{NH}_{2} \mathrm{CONH}$ species was identified as the potential-limiting step, and the corresponding $U_{\mathrm{L}}^{\text {urea }}$ were computed to be $-0.49,-0.65$, and $-0.52 \mathrm{~V}$ for $\mathrm{Mo}_{2} \mathrm{~B}_{2}, \mathrm{Ti}_{2} \mathrm{~B}_{2}$, $\mathrm{Cr}_{2} \mathrm{~B}_{2}$, respectively. Remarkably, the $U_{\mathrm{L}}^{\text {urea }}$ of three MBenes are comparable to or even lower than that of the $\mathrm{Pd}-\mathrm{Cu}$ catalyst $(-0.64 \mathrm{~V})$, which is indicative of superior electrocatalytic reactivity toward urea formation. Moreover, the adsorption energies of ${ }^{*} \mathrm{NH}_{2} \mathrm{CONH}_{2}$ on $2 \mathrm{D} \mathrm{Mo}_{2} \mathrm{~B}_{2}, \mathrm{Ti}_{2} \mathrm{~B}_{2}$, and $\mathrm{Cr}_{2} \mathrm{~B}_{2}$ are $-1.28,-1.55$, and $-1.21 \mathrm{eV}$, respectively, which are lower than that of the $\mathrm{Pd}-\mathrm{Cu}$ catalyst $(-1.68 \mathrm{eV})^{23}$, indicating that the formed urea molecule can be easily released, especially when electrochemical reactions are carried out in flow cells.

For computational simplicity, the above results on electrochemical steps were all obtained by assuming that the catalyst is charge neutral, and thus, the Fermi-level of the catalyst would change with the variation in adsorbed reaction species. However, in real electrochemical reactions, electron transfer between the catalyst and the electrode to match the Fermi level of the catalyst with the applied electrode potential. In a recent theoretical study, Kim et al. ${ }^{40}$ revealed that the surface charge can have a substantial effect on the electrochemical activity of graphene-based materials. To this end, we also performed grand-canonical DFT computations to investigate the effect of surface charge on the electrocatalytic reactivity of the three MBenes toward urea formation. Supplementary Fig. 6 presents the free energy of all reaction intermediates of urea formation computed using the constant-potential method (CPM), and those computed from the constant-charge method (CCM) are also listed for comparison. Remarkably, the energy difference between the two methods is within the range of $0.1 \mathrm{eV}$ for all three MBenes, which is similar to the values found in bulk materials but much lower than that of graphene. The less pronounced charge effect on MBenes in comparison to graphene should be attributed to greater thickness.

\section{Discussion}

Electrocatalytic selectivity of $\mathrm{CO}_{2}$ reduction on MBenes. The above results demonstrated that the electrochemical production of urea could be feasible on the surfaces of $2 \mathrm{D} \mathrm{Mo} \mathrm{B}_{2}, \mathrm{Ti}_{2} \mathrm{~B}_{2}$, and $\mathrm{Cr}_{2} \mathrm{~B}_{2}$. However, at present we still cannot claim that these three 2D MBenes are qualified catalysts for urea production as some important issues concerning the selectivity are still pending. First, as we discussed above, the ${ }^{*} \mathrm{CO}$ species is the key intermediate for the entire reaction. Although we demonstrated that the formation of $* \mathrm{CO}$ on the surfaces of the three studied MBenes is feasible via the intermediate ${ }^{*} \mathrm{COOH}$, the transfer of a first proton/electron pair to $\mathrm{CO}_{2}$ could also lead to the formation of $* \mathrm{OCHO}$, which is 

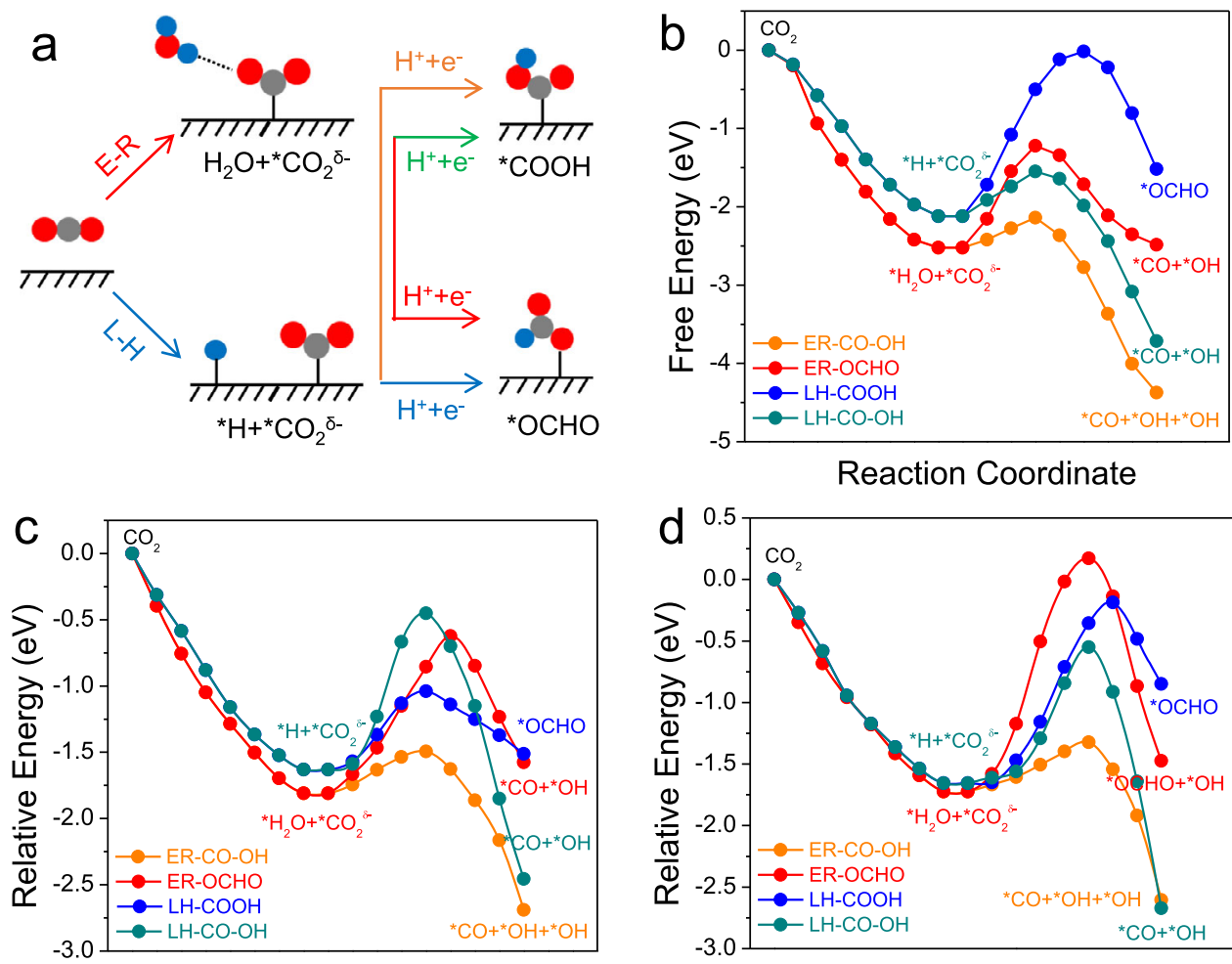

Reaction Coordinate

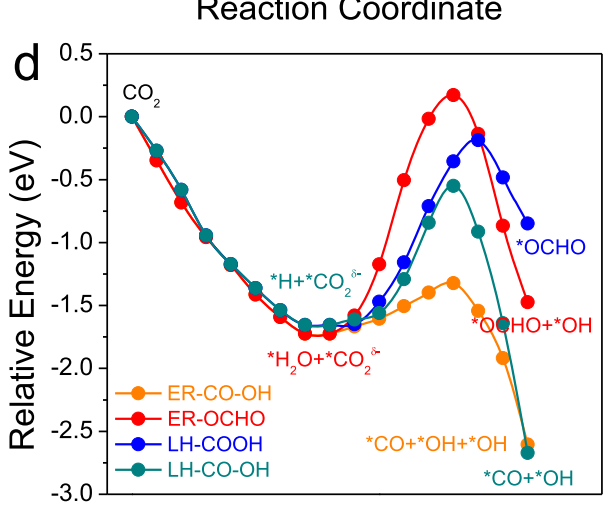

Reaction Coordinate

Fig. 4 Selectivity of $\mathrm{CO}_{2}$ electroreduction. a Schematic diagram of the ER and $\mathrm{LH}$ mechanisms of $\mathrm{CO}_{2}$ electroreduction to ${ }^{\star} \mathrm{COOH}$ or ${ }^{\star} \mathrm{OCHO}$. Kinetic pathways for the electroreduction of $\mathrm{CO}_{2}$ on the surfaces of $(\mathbf{b}) \mathrm{Mo}_{2} \mathrm{~B}_{2}, \mathbf{c} \mathrm{Ti}_{2} \mathrm{~B}_{2}$, and $\mathbf{d} \mathrm{Cr}_{2} \mathrm{~B}_{2}$.

a key intermediate of formate. Therefore, knowing whether $\mathrm{CO}_{2}$ can be selectively reduced to ${ }^{*} \mathrm{CO}$ is very important. To address this concern, we first computed the binding energy of the * OCHO species on three MBenes, and found that the binding energy of ${ }^{*} \mathrm{OCHO}$ is generally higher than the binding energy of ${ }^{*} \mathrm{COOH}$. However, this does not mean that the formation of ${ }^{*} \mathrm{OCHO}$ is preferred. As revealed by previous studies ${ }^{41,42}$, many transition metal catalysts bind the * $\mathrm{OCHO}$ species more strongly than the ${ }^{*} \mathrm{COOH}$ species, but they all have $\mathrm{CO}$, rather than formate, as the major product. Therefore, predicting the selectivity of ${ }^{*} \mathrm{CO}$ formation solely based on thermodynamics computations is actually not reliable.

As revealed by Cheng et al. ${ }^{43}$, the selectivity of $\mathrm{CO}_{2}$ reduction is essentially controlled kinetically, and the formation of ${ }^{*} \mathrm{COOH}$ and ${ }^{*} \mathrm{OCHO}$ can be achieved via either the Eley-Raideal (ER) mechanism by accepting a $\mathrm{H}$ atom from water or via the Langmuir-Hinshelwood (LH) mechanism by accepting a surfacebound $\mathrm{H}$ atom (Fig. 4a). To obtain some deep insight into the selectivity, we also constructed explicit liquid/solid interfaces for all three MBenes by adding a water layer on their surfaces (Supplementary Fig. 7), and both the ER and LH mechanisms were considered for the formation of ${ }^{*} \mathrm{COOH}$ and ${ }^{*} \mathrm{OCHO}$. As shown in Fig. $4 \mathrm{~b}-\mathrm{d}$, for the formation of the ${ }^{*} \mathrm{COOH}$ species, the ER mechanism is preferred with kinetic barriers of $0.38,0.31$, and $0.32 \mathrm{eV}$ for the $2 \mathrm{D} \mathrm{Mo}_{2} \mathrm{~B}_{2}, \mathrm{Ti}_{2} \mathrm{~B}_{2}$, and $\mathrm{Cr}_{2} \mathrm{~B}_{2}$, respectively. In contrast, the formation of the ${ }^{*} \mathrm{OCHO}$ species entails much large kinetic barrier via either the ER mechanism or the LH mechanism. Therefore, on the surfaces of our three chosen 2D MBenes, $\mathrm{CO}_{2}$ would be dominantly reduced to ${ }^{*} \mathrm{CO}$ rather than formate.

The second concern regarding the ${ }^{*} \mathrm{CO}$ species is whether it could be further reduced to ${ }^{*} \mathrm{CHO}$ or ${ }^{*} \mathrm{COH}$ under the working conditions of urea production. To address this question, we also computed the free energy of ${ }^{*} \mathrm{CO}$ reduction to ${ }^{*} \mathrm{CHO}$ and ${ }^{*} \mathrm{COH}$ for $2 \mathrm{D} \mathrm{Mo}_{2} \mathrm{~B}_{2}, \mathrm{Ti}_{2} \mathrm{~B}_{2}$, and $\mathrm{Cr}_{2} \mathrm{~B}_{2}$ (Supplementary Table 4). The limiting potential for the formation of ${ }^{*} \mathrm{CHO}$ or $* \mathrm{COH}$ on the three MBenes is at least $-1.22 \mathrm{~V}$, which is much higher than the $U_{\mathrm{L}}^{\text {urea }}$. Therefore, the formation of ${ }^{*} \mathrm{CHO}$ or ${ }^{*} \mathrm{COH}$ would be significantly suppressed under the working potential of urea production.

Moreover, we are also aware of that there would be competition between ${ }^{*} \mathrm{CO}$ adsorption and adsorption/dissociation of $\mathrm{H}_{2} \mathrm{O}$ molecule in the realistic aqueous environment. According to our results, the kinetic barrier for the direct dissociation of one $\mathrm{H}_{2} \mathrm{O}$ molecule on $2 \mathrm{D} \mathrm{Mo}_{2} \mathrm{~B}_{2}, \mathrm{Ti}_{2} \mathrm{~B}_{2}$, and $\mathrm{Cr}_{2} \mathrm{~B}_{2}$ is $0.63,0.53$, and $0.72 \mathrm{eV}$, respectively (Supplementary Fig. 8), indicating the formation of surface bounded ${ }^{*} \mathrm{H}$ and ${ }^{*} \mathrm{OH}$ is also feasible for three MBenes. While the ${ }^{*} \mathrm{H}$ species can serve as the proton source to react with the reaction intermediates via the $\mathrm{LH}$ mechanism, there could exist the adsorption competition between ${ }^{*} \mathrm{CO}$ and ${ }^{*} \mathrm{OH}$ species on three MBenes. Therefore, we then plotted the curves of equilibrium surface coverages of these two species as a function of electrode potential by performing microkinetic simulations. As shown in Supplementary Fig. 9, the population of ${ }^{*} \mathrm{OH}$ and ${ }^{*} \mathrm{CO}$ is potential dependent. Specifically, the surfaces of three MBenes would be predominately covered by $\mathrm{OH}^{*}$ species under low electrode potential. When the electrode potential is higher than $-0.31,-0.64$, and $-0.22 \mathrm{~V}$, respectively, the coverage of $\mathrm{CO}^{*}$ on $2 \mathrm{D} \mathrm{Mo}_{2} \mathrm{~B}_{2}, \mathrm{Ti}_{2} \mathrm{~B}_{2}$ and $\mathrm{Cr}_{2} \mathrm{~B}_{2}$ begins to increase, while the coverage of $\mathrm{OH}^{*}$ begins to decrease. Since the critical potential of ${ }^{*} \mathrm{CO} /{ }^{*} \mathrm{OH}$ adsorption for each MBene is lower than the respective $U_{\mathrm{L}}^{\text {urea }}$, it can be expected that surface active sites of $2 \mathrm{D}$ MBenes, especially $\mathrm{Mo}_{2} \mathrm{~B}_{2}$ and $\mathrm{Cr}_{2} \mathrm{~B}_{2}$, would be mainly covered by ${ }^{*} \mathrm{CO}$ rather than ${ }^{*} \mathrm{OH}$ under working potentials.

Electrocatalytic selectivity of $\mathrm{N}_{2}$ reduction on MBenes. As reported by previous studies ${ }^{37,38}$, many $2 \mathrm{D}$ MBenes have basal 

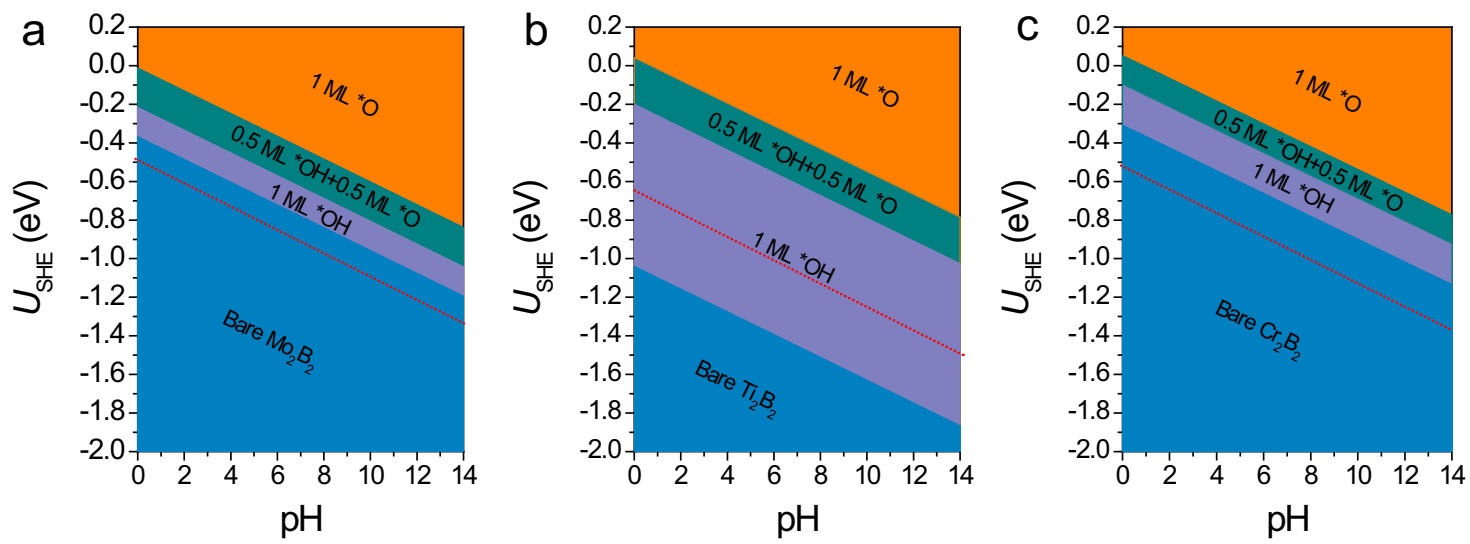

Fig. 5 Surface states of MBenes under electrochemical conditions. Surface Pourbaix diagrams of $2 \mathrm{D}(\mathbf{a}) \mathrm{Mo}_{2} \mathrm{~B}_{2},(\mathbf{b}) \mathrm{Ti}_{2} \mathrm{~B}_{2}$, and $(\mathbf{c}) \mathrm{Cr}_{2} \mathrm{~B}_{2}$. The thermodynamically stable states of the surface under $\mathrm{SHE}$ and $\mathrm{pH}$ values are highlighted by orange (for $\left.{ }^{\star} \mathrm{O}\right)$, green $\left(\right.$ for $\left.{ }^{\star} \mathrm{O}+{ }^{\star} \mathrm{OH}\right)$, and purple (for ${ }^{\star} \mathrm{OH}$ ). The red dashed line represents the limiting potential of urea formation.
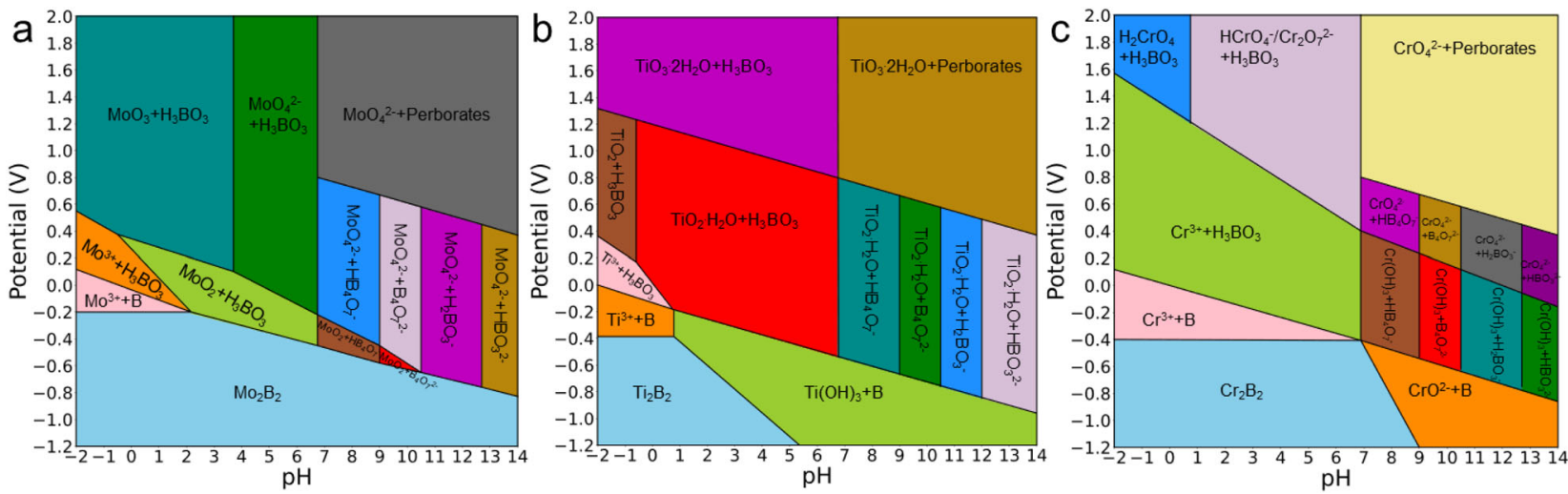

Fig. 6 Corrosion resistance of MBenes under electrochemical conditions. Computationally predicted Pourbaix diagrams of $(\mathbf{a}) \mathrm{Mo}_{2} \mathrm{~B}_{2}$, (b) $\mathrm{Ti}_{2} \mathrm{~B}_{2}$, and $(\mathbf{c})$ $\mathrm{Cr}_{2} \mathrm{~B}_{2}$ using $10^{-6} \mathrm{M}$ concentration for aqueous species at $25^{\circ} \mathrm{C}$.

plane activity for $\mathrm{N}_{2}$ electroreduction to $\mathrm{NH}_{3}$. Would the adsorbed $\mathrm{N}_{2}$ molecule also be reduced to $\mathrm{NH}_{3}$ on our three chosen $\mathrm{M}_{2} \mathrm{~B}_{2}$-type MBenes? To address this question, we investigated the thermodynamics of the electrochemical NRR on 2D $\mathrm{Mo}_{2} \mathrm{~B}_{2}, \mathrm{Ti}_{2} \mathrm{~B}_{2}, \mathrm{Cr}_{2} \mathrm{~B}_{2}$ to get some deep insight. As shown in Supplementary Fig. 10, the electrochemical NRR on all three $\mathrm{M}_{2} \mathrm{~B}_{2}$ monolayers is feasible, with $U_{\mathrm{L}}^{\mathrm{NH}_{3}}$ values of $-0.79,-0.71$, and $-0.65 \mathrm{~V}$, respectively. Encouragingly, for all three MBenes, $U_{\mathrm{L}}^{\mathrm{NH}_{3}}$ is higher than $U_{\mathrm{L}}^{\text {urea }}$, suggesting that the formation of the $\mathrm{NH}_{3}$ can be greatly suppressed on these three MBenes.

Since kinetic factors play an important role in determining the selectivity, we further computed the kinetic barriers of elementary steps of $\mathrm{N}_{2}$ reduction to urea for three 2D MBenes and compared with those of $\mathrm{N}_{2}$ reduction $\mathrm{NH}_{3}$. Especially, both ER and $\mathrm{LH}$ mechanisms were considered for the electrochemical steps. As presented in Supplementary Figs. 11-13, for all three 2D MBenes, the non-electrochemical step of ${ }^{*} \mathrm{NCON}$ formation has the biggest kinetic barrier, which is $0.53,0.77$, and $0.70 \mathrm{eV}$ for $\mathrm{Mo}_{2} \mathrm{~B}_{2}$, $\mathrm{Ti}_{2} \mathrm{~B}_{2}, \mathrm{Cr}_{2} \mathrm{~B}_{2}$, respectively. Note that the kinetic barriers of $*$ NCON formation predicted from explicit solvent model are quite close to those predicted from implicit solvent model. As a comparison, the first electrochemical step of $\mathrm{N}_{2}$ reduction to $\mathrm{NH}_{3}$, namely $\mathrm{N}_{2}$ reduction to $\mathrm{NNH}$, already has a relatively big kinetic barrier, which is $0.78,0.80$, and 0.76 for $2 \mathrm{D} \mathrm{Mo}_{2} \mathrm{~B}_{2}, \mathrm{Ti}_{2} \mathrm{~B}_{2}$, $\mathrm{Cr}_{2} \mathrm{~B}_{2}$, respectively (Supplementary Fig. 14). Therefore, the urea synthesis is also kinetically favorable on these three $2 \mathrm{D}$ MBenes.
Pourbaix diagrams of MBenes. Finally, another concern that also needs to be addressed is the electrochemical stability of the three MBenes. Although no evidence yet confirm the existence of functional groups on experimentally realized MBenes, we wondered whether the bare surfaces of MBenes could be covered by $* \mathrm{O} /{ }^{*} \mathrm{OH}$ species in aqueous solution under working conditions. To answer this question, we constructed surface Pourbaix diagrams of the three MBenes to reveal the most stable surface configurations under different equilibrium potentials and $\mathrm{pH}$ values (the computational details are given in Supplementary Methods $)^{44,45}$. As shown in Fig. 5, when the electrode potential is $0 \mathrm{~V}$ vs SHE, the basal plane of the $2 \mathrm{D} \mathrm{Mo}_{2} \mathrm{~B}_{2}$ is fully covered by * $\mathrm{O}$ species independent of the $\mathrm{pH}$ value, whereas the basal planes of the $2 \mathrm{D} \mathrm{Ti}_{2} \mathrm{~B}_{2}$ and $\mathrm{Cr}_{2} \mathrm{~B}_{2}$ are covered by $* \mathrm{O}$ and $* \mathrm{OH}$ groups in a strong acid environment. When an electrode potential is applied, the hydrogenation of $* \mathrm{O}$ and $* \mathrm{OH}$ becomes energetically favorable on the surfaces of all three MBenes. In particular, the minimum potentials required to remove the surface ${ }^{*} \mathrm{O} /{ }^{*} \mathrm{OH}$ species at $\mathrm{pH}=0\left(U_{\mathrm{R}}\right)$ are $-0.37,-1.04$, and $-0.31 \mathrm{~V}$ for the $2 \mathrm{D}$ $\mathrm{Mo}_{2} \mathrm{~B}_{2}, \mathrm{Ti}_{2} \mathrm{~B}_{2}$, and $\mathrm{Cr}_{2} \mathrm{~B}_{2}$ monolayers, respectively. It is worth noting that the $U_{R}$ of $2 \mathrm{D} \mathrm{Mo} \mathrm{B}_{2}$ and $\mathrm{Cr}_{2} \mathrm{~B}_{2}$ is less negative than the respective $U_{\mathrm{L}}^{\mathrm{urea}}$, indicating that these two MBenes could possess superior electrochemical stability against surface oxidation under working conditions. In sharp contrast, the $U_{R}$ of $2 \mathrm{D}$ $\mathrm{Ti}_{2} \mathrm{~B}_{2}$ is far more negative than its $U_{\mathrm{L}}^{\text {urea }}$ and even $U_{\mathrm{L}}^{\mathrm{NH}_{3}}$. As the electrode potential of urea production on the $2 \mathrm{D} \mathrm{Ti}_{2} \mathrm{~B}_{2}$ should not exceed the $U_{\mathrm{L}}^{\mathrm{NH}_{3}}$ to guarantee a high selectivity, the surface of the 
$2 \mathrm{D} \mathrm{Ti}{ }_{2} \mathrm{~B}_{2}$ would inevitably be occupied by $* \mathrm{OH} /{ }^{*} \mathrm{O}$ species under working conditions, resulting in decreased reactive sites on the basal plane.

Besides the stability of active surfaces, whether the catalyst itself would corrode under electrochemical conditions also needs to be addressed ${ }^{46,47}$. Taking advantage of the fact that the formation energies are transferable between energy reference systems, we further plotted the whole Pourbaix diagram of the three $2 \mathrm{D}$ MBenes as a function of $\mathrm{pH}$ and potential at standard conditions to identified their stability window in aqueous solutions by directly combining Gibbs free energies from DFT computations with experimental arbitrary aqueous states. As shown in Fig. 6, a wide passivation region can be identified for $\mathrm{Mo}_{2} \mathrm{~B}_{2} \quad(\mathrm{pH}<7.08)$ and $\mathrm{Cr}_{2} \mathrm{~B}_{2}(\mathrm{pH}<7.22)$ at the potential of $-0.49 \mathrm{~V}$ and $-0.52 \mathrm{~V}$, respectively. Using the electrolyte of $\mathrm{Pd}-\mathrm{Cu}$ catalyst $(\mathrm{pH}=6.8)^{23}$ as a reference, both $2 \mathrm{D} \mathrm{Mo}_{2} \mathrm{~B}_{2}$ and $\mathrm{Cr}_{2} \mathrm{~B}_{2}$ can maintain structure integrality under working conditions due to high barriers for solid-liquid phase transformations. In sharp contrast to $2 \mathrm{D} \mathrm{Mo}_{2} \mathrm{~B}_{2}$ and $\mathrm{Cr}_{2} \mathrm{~B}_{2}, 2 \mathrm{D} \mathrm{Ti} \mathrm{B}_{2}$ has a narrow passivation region $(\mathrm{pH}<2.26)$ when subjected to an external potential of $-0.65 \mathrm{~V}$. At high $\mathrm{pH}$ region, $\mathrm{Ti}_{2} \mathrm{~B}_{2}$ would be transformed into $\mathrm{Ti}(\mathrm{OH})_{3}$, indicating that $\mathrm{Ti}_{2} \mathrm{~B}_{2}$ would be easily corroded under working conditions of urea synthesis, Therefore, $2 \mathrm{D} \mathrm{Ti}{ }_{2} \mathrm{~B}_{2}$ is not a qualified electrocatalyst for urea formation due to its low electrochemical stability.

Activity origin of MBenes toward urea production. Due to the good reactivity, selectivity, and stability, $2 \mathrm{D} \mathrm{Mo}_{2} \mathrm{~B}_{2}$ and $\mathrm{Cr}_{2} \mathrm{~B}_{2}$ have been identified as promising electrocatalysts for urea synthesis. However, at present, the activity origin of these two MBenes is not yet clear. It is known that the activity of an electrocatalyst is essentially governed by its electronic structure. Therefore, in order to get some deeper insights into the specialty of MBenes for urea synthesis, we further computed their electronic density of states (DOS) at the Fermi level and compared with those of some other experimentally realized $2 \mathrm{D}$ metallic materials, including four bare MXenes $\left(\mathrm{Ti}_{2} \mathrm{C}, \mathrm{Mo}_{2} \mathrm{C}, \mathrm{Ti}_{3} \mathrm{C}_{2}\right.$, $\left.\mathrm{Mo}_{3} \mathrm{C}_{2}\right)$ and two transition metal chalcogenides $\left(1 \mathrm{~T}-\mathrm{MoS}_{2}, \mathrm{VS}_{2}\right)$. As shown in Supplementary Fig. 15, the DOS per atom of MXenes are generally much higher than those of $2 \mathrm{D} \mathrm{Mo}_{2} \mathrm{~B}_{2}$ and $\mathrm{Cr}_{2} \mathrm{~B}_{2}$, implying stronger metallicity and more active surfaces of MXenes. However, this does not mean that MXenes have better electrochemical activity than MBenes, because the active surfaces of MXenes are known to be easily passivated in solvents ${ }^{27-29}$. Interestingly, the DOS of $\mathrm{Ti}_{2} \mathrm{~B}_{2}$ are quite close to those of $\mathrm{Mo}_{2} \mathrm{C}$ and $\mathrm{Mo}_{3} \mathrm{C}_{2}$, which could explain the instability of $\mathrm{Ti}_{2} \mathrm{~B}_{2}$ in aqueous solution. Remarkably, the DOS of $\mathrm{Mo}_{2} \mathrm{~B}_{2}$ and $\mathrm{Cr}_{2} \mathrm{~B}_{2}$ are significantly higher than those of $1 \mathrm{~T}-\mathrm{MoS}_{2}$ and $\mathrm{VS}_{2}$ that are actually inert to urea production according to our test computations. Therefore, the good activity and stability of $2 \mathrm{D} \mathrm{Mo}_{2} \mathrm{~B}_{2}$ and $\mathrm{Cr}_{2} \mathrm{~B}_{2}$ should be attributed to their moderate metallicity.

To summarize, on the basis of comprehensive DFT computations, we have systematically explored the potential of utilizing the three experimentally realized MBenes, $\mathrm{Mo}_{2} \mathrm{~B}_{2}, \mathrm{Ti}_{2} \mathrm{~B}_{2}$, and $\mathrm{Cr}_{2} \mathrm{~B}_{2}$, as electrocatalysts for urea synthesis. The activity, selectivity, and stability of the three MBenes under aqueous conditions were carefully studied. Our computations demonstrated that all three MBenes can adsorb $\mathrm{N}_{2}$ and $\mathrm{CO}_{2}$ on their basal planes, and the adsorbed $\mathrm{CO}_{2}$ can be easily reduced to $* \mathrm{CO}$. Afterwards, the key intermediate ${ }^{*} \mathrm{NCON}$ can be formed via the coupling of ${ }^{*} \mathrm{~N}_{2}$ and ${ }^{*} \mathrm{CO}$, which can be further reduced to urea via four PCET steps. The limiting potentials of urea formation for our three studied MBenes are in the range of -0.49 to $-0.65 \mathrm{eV}$, which are comparable to that of the $\mathrm{Pd}-\mathrm{Cu}$ alloy catalyst. In particular, it is found that $2 \mathrm{D} \mathrm{Mo}_{2} \mathrm{~B}_{2}$ and $\mathrm{Cr}_{2} \mathrm{~B}_{2}$ can prevent the problems of active sites blockage and self-corrosion, while 2D $\mathrm{Ti}_{2} \mathrm{~B}_{2}$ not only has its surface active sites occupied by ${ }^{*} \mathrm{OH}$ and ${ }^{*} \mathrm{O}$ groups, but also could be easily corroded under reaction conditions. Therefore, 2D $\mathrm{Mo}_{2} \mathrm{~B}_{2}$ and $\mathrm{Cr}_{2} \mathrm{~B}_{2}$ can serve as promising catalysts for urea production, which can be attributed to their moderate metallicity. Our work provides a clear roadmap for the design of electrocatalysts for simultaneously fixing $\mathrm{N}_{2}$ and $\mathrm{CO}_{2}$ to produce urea, which could promote more experimental and theoretical efforts on developing $2 \mathrm{D}$ electrocatalysts for this challenging reaction.

\section{Methods}

DFT computations. Our DFT computations based on first-principles were performed via the Vienna ab initio simulation package (VASP) ${ }^{48}$. The ion-electron interactions were described with the projector-augmented plane-wave (PAW) method $^{49}$. Exchange-correlation potentials were expressed by

Perdew-Burke-Ernzerhof (PBE) functional with the generalized gradient approximation (GGA) ${ }^{50}$. A $460 \mathrm{eV}$ cutoff energy for the plane wave expansion was adopted in all the computations. A Monkhorst-Pack k-points setting of $3 \times 3 \times 1$ and $15 \times 15 \times 1$ was used to sample the $2 \mathrm{D}$ Brillouin zone for geometry optimizations and electronic structure computations, respectively. We set the $x$ and $y$ directions parallel and the $z$ direction perpendicular to the layer plane, and adopted a vacuum layer length of $20 \AA$ in the $z$ direction. The systems were relaxed until the energy and force reaching the convergence threshold of $10^{-5} \mathrm{eV}$ and $0.01 \mathrm{eV} / \mathrm{A}$.

The phonon spectra were computed using the density functional perturbation theory (DFPT), as implemented in the Phonon code $\mathrm{e}^{51}$. The ab initio molecular dynamic (AIMD) simulations were performed using the NVT ensemble. The NoséHoover method simulations last $10 \mathrm{ps}$ with a time step of $1.0 \mathrm{fs}^{52}$. The solvation effects in aqueous solution were considered with the Poisson-Boltzmann implicit solvation model as implemented in VASP (VASP-sol), where the dielectric constant of water was taken as $80^{53}$. The climbing-image nudged elastic band (CINEB) method ${ }^{54}$ as implemented in VASP was used to obtain the kinetic barriers.

The grand-canonical DFT computations, which allow the number of electrons to adjust automatically at a fixed electron chemical potential, were performed using the PBE functional as implemented in JDFTx code ${ }^{55}$ with a cutoff energy of 20 Hartree. The JDFTx code combines electronic DFT with classical DFT and continuum models of liquids for first-principles computations of electrochemical systems. The charge-asymmetric nonlocal determined local electric (CANDLE) 56 solvation model as implemented in JDFTx was utilized for describing the electrolyte. Other numerical parameters, including k-point sampling, convergence criteria, etc. are similar to the VASP computations.

Free energy computations. To compute the free energy change $(\Delta G)$ of each elementary step of electrochemical urea synthesis, we adopted the computational hydrogen electrode (CHE) model developed by Nørskov et al. ${ }^{57,58}$ according to which the $\Delta G$ of an electrochemical reaction is computed as:

$$
\Delta G=\Delta E+\Delta E_{\mathrm{ZPE}}-T \Delta S
$$

where $\Delta E$ is the DFT computed reaction energy, $\Delta E_{\mathrm{ZPE}}$ and $\Delta S$ are the zero-point energy difference and the entropy difference between the adsorbed state and the gas phase, respectively, and $T$ is the temperature ( $298.15 \mathrm{~K}$, in our work). For adsorbed reaction intermediates, their $E_{\mathrm{ZPE}}$ and $S$ are obtained via vibrational frequencies computations with harmonic approximation and neglecting contributions from the slab, while for molecules these are taken from the NIST database. Moreover, in accordance with the CHE model, the effects of electrode potential $(U)$ and $\mathrm{pH}$ can be treated as an energy shift to free energy change in the electrochemical steps:

$$
\begin{gathered}
\Delta G_{U}=-e U \\
\Delta G_{\mathrm{pH}}=-k_{B} T \ln 10 \times \mathrm{pH}
\end{gathered}
$$

where $k_{\mathrm{B}}$ is Boltzmann constant. In this work, the value of $\mathrm{pH}$ was assumed to be zero in free energy computations.

\section{Data availability}

The authors declare that the data supporting the findings of this study are available within the paper and its supplementary information files. All of the other data are available from the corresponding author upon reasonable request.

\section{Code availability}

The computational codes used in this work are available from the corresponding author on reasonable request.

Received: 16 February 2021; Accepted: 11 June 2021; Published online: 02 July 2021 


\section{References}

1. Comer, B. M. et al. Prospects and challenges for solar fertilizers. Joule 3, 1578-1605 (2019).

2. Liu, Y., Zhao, X. \& Ye, L. A novel elastic urea-melamine-formaldehyde foam: structure and properties. Ind. Eng. Chem. Res. 55, 8743-8750 (2016).

3. Celleno, L. Topical urea in skincare: a review. Dermatol. Ther. 31, e12690 (2018).

4. Huang, H. -M., McDouall, J. J. W. \& Procter, D. J. Radical anions from ureatype carbonyls: radical cyclizations and cyclization cascades. Angew. Chem. Int. Ed. 57, 4995-4999 (2018).

5. Seneque, M., Can, F., Duprez, D. \& Courtois, X. $\mathrm{NO}_{\mathrm{x}}$ selective catalytic reduction $\left(\mathrm{NO}_{\mathrm{x}}\right.$-SCR) by urea: evidence of the reactivity of $\mathrm{HNCO}$, including a specific reaction pathway for $\mathrm{NO}_{\mathrm{x}}$ reduction involving $\mathrm{NO}+\mathrm{NO}_{2}$. ACS Catal. 6, 4064-4067 (2016).

6. Barzagli, F., Mani, F. \& Peruzzini, M. From greenhouse gas to feedstock: formation of ammonium carbamate from $\mathrm{CO}_{2}$ and $\mathrm{NH}_{3}$ in organic solvents and its catalytic conversion into urea under mild conditions. Green. Chem. 13, 1267-1274 (2011).

7. Pérez-Fortes, M., Bocin-Dumitriu, A. \& Tzimas, E. $\mathrm{CO}_{2}$ utilization pathways: techno-economic assessment and market opportunities. Energy Procedia 63, 7968-7975 (2014).

8. Erisman, J. W., Sutton, M. A., Galloway, J., Klimont, Z. \& Winiwarter, W. How a century of ammonia synthesis changed the world. Nat. Geosci. 1, 636-639 (2008).

9. Service, R. F. New recipe produces ammonia from air, water, and sunlight. Science 345, 610 (2014).

10. Licht, $\mathrm{S}$. et al. Ammonia synthesis by $\mathrm{N}_{2}$ and steam electrolysis in molten hydyoxide suspensions of nanoscale $\mathrm{Fe}_{2} \mathrm{O}_{3}$. Science 345, 637-640 (2014).

11. Qian, J., An, Q., Fortunelli, A., Nielsen, R. J. \& Goddard, W. A. Reaction mechanism and kinetics for ammonia synthesis on the Fe(111) surface. J. Am. Chem. Soc. 140, 6288-6297 (2018).

12. Zheng, J. et al. Photoelectrochemical synthesis of ammonia on the aerophilichydrophilic heterostructure with $37.8 \%$ efficiency. Chemistry 5, 617-633 (2019).

13. Li, H., Shang, J., Ai, Z. \& Zhang, L. Efficient visible light nitrogen fixation with $\mathrm{BiOBr}$ nanosheets of oxygen vacancies on the exposed (001) facets. J. Am. Chem. Soc. 137, 6393-6399 (2015).

14. Foster, S. L. et al. Catalysts for nitrogen reduction to ammonia. Nat. Catal. 1, 490-500 (2018).

15. Wan, Y., Xu, J. \& Lv, R. Heterogeneous electrocatalysts design for nitrogen reduction reaction under ambient conditions. Mater. Today 27, 69-90 (2019).

16. Montoya, J. H., Tsai, C., Vojvodic, A. \& Nørskov, J. K. The challenge of electrochemical ammonia synthesis: a new perspective on the role of nitrogen scaling relations. ChemSusChem 8, 2180-2186 (2015).

17. Ling, C. et al. New mechanism for $\mathrm{N}_{2}$ reduction: the essential role of surface hydrogenation. J. Am. Chem. Soc. 141, 18264-18270 (2019).

18. Qing, $\mathrm{G}$. et al. Recent advances and challenges of electrocatalytic $\mathrm{N}_{2}$ reduction to ammonia. Chem. Rev. 120, 5437-5516 (2020).

19. Suryanto, B. H. R. et al. Challenges and prospects in the catalysis of electroreduction of nitrogen to ammonia. Nat. Catal. 2, 290-296 (2019).

20. Liu, H. et al. Homogeneous, heterogeneous, and biological catalysts for electrochemical $\mathrm{N}_{2}$ reduction toward $\mathrm{NH}_{3}$ under ambient conditions. ACS Catal. 9, 5245-5267 (2019).

21. Jouny, M. et al. Formation of carbon-nitrogen bonds in carbon monoxide electrolysis. Nat. Chem. 11, 846-851 (2019).

22. Comer, B. M. et al. The role of adventitious carbon in photo-catalytic nitrogen fixation by titania. J. Am. Chem. Soc. 140, 15157-15160 (2018).

23. Chen, C. et al. Coupling $\mathrm{N}_{2}$ and $\mathrm{CO}_{2}$ in $\mathrm{H}_{2} \mathrm{O}$ to synthesize urea under ambient conditions. Nat. Chem. 12, 717-724 (2020).

24. Sun, Z., Ma, T., Tao, H., Fan, Q. \& Han, B. Fundamentals and challenges of electrochemical $\mathrm{CO}_{2}$ reduction using two-dimensional materials. Chemistry 3 , 560-587 (2017).

25. Zhu, X. \& Li, Y. Review of two-dimensional materials for electrochemical $\mathrm{CO}_{2}$ reduction from a theoretical perspective. Wiley Interdiscip. Rev. Comput. Mol. Sci. 9, e1416 (2019).

26. $\mathrm{Li}, \mathrm{N}$. et al. Understanding of electrochemical mechanisms for $\mathrm{CO}_{2}$ capture and conversion into hydrocarbon fuels in transition-metal carbides (MXenes). ACS Nano 11, 10825-10833 (2017).

27. Gogotsi, Y. \& Anasori, B. The rise of MXenes. ACS Nano 13, 8491-8494 (2019).

28. Shahzad, F., Iqbal, A., Kim, H. \& Koo, C. M. 2D transition metal carbides (MXenes): applications as an electrically conducting material. Adv. Mater. 32, 2002159 (2020).

29. Pang, J. et al. Applications of 2D MXenes in energy conversion and storage systems. Chem. Soc. Rev. 48, 72-123 (2019).

30. Zhang, H., Xiang, H., Dai, F. Z., Zhang, Z. \& Zhou, Y. First demonstration of possible two-dimensional MBene $\mathrm{CrB}$ derived from $\mathrm{MAB}$ phase $\mathrm{Cr}_{2} \mathrm{AlB}_{2}$. J. Mater. Sci. Technol. 34, 2022-2026 (2018).
31. Kota, S. et al. Magnetic properties of $\mathrm{Cr}_{2} \mathrm{AlB}_{2}, \mathrm{Cr}_{3} \mathrm{AlB}_{4}$, and $\mathrm{CrB}$ powders. $J$. Alloy. Compd. 767, 474-482 (2018).

32. Wang, J. et al. Discovery of hexagonal ternary phase $\mathrm{Ti}_{2} \mathrm{InB}_{2}$ and its evolution to layered boride TiB. Nat. Commun. 10, 1-8 (2019).

33. Alameda, L. T. et al. Multi-step topochemical pathway to metastable $\mathrm{Mo}_{2} \mathrm{AlB}_{2}$ and related two-dimensional nanosheet heterostructures. J. Am. Chem. Soc. 141, 10852-10861 (2019).

34. Zhang, T., Zhang, B., Peng, Q., Zhou, J. \& Sun, Z. $\mathrm{Mo}_{2} \mathrm{~B}_{2}$ MBene-supported single-atom catalysts as bifunctional HER/OER and OER/ORR electrocatalysts. J. Mater. Chem. A 9, 433-441 (2021).

35. Rezaie, A. A. et al. Synthesis and Li-ion electrode properties of layered MAB phases $\mathrm{Ni}_{\mathrm{n}+1} \mathrm{ZnB}_{\mathrm{n}}(\mathrm{n}=1,2)$. J. Mater. Chem. A 8, 1646-1651 (2020).

36. Siriwardane, E. M. D., Joshi, R. P., Kumar, N. \& Çaklr, D. Revealing the Formation energy-exfoliation energy-structure correlation of MAB phases using machine learning and DFT. ACS Appl. Mater. Interfaces 12, 29424-29431 (2020).

37. Yang, X., Shang, C., Zhou, S. \& Zhao, J. MBenes: emerging 2D materials as efficient electrocatalysts for the nitrogen reduction reaction. Nanoscale Horiz. 5, 1106-1115 (2020).

38. Guo, X. et al. Establishing a theoretical landscape for identifying basal plane active 2D metal borides (MBenes) toward nitrogen electroreduction. Adv. Funct. Mater. 31, 2008056 (2021).

39. Born, M.; Huang, H. Dynamical theory of crystal lattices, Clarendon, Oxford, 1954.

40. Kim, D., Shi, J. \& Liu, Y. Substantial impact of charge on electrochemical reactions of two-dimensional materials. J. Am. Chem. Soc. 140, 9127-9131 (2018).

41. Feaster, J. T. et al. Understanding selectivity for the electrochemical reduction of carbon dioxide to formic acid and carbon monoxide on metal electrodes. ACS Catal. 7, 4822-4827 (2017).

42. Yoo, J. S., Christensen, R., Vegge, T., Nørskov, J. K. \& Studt, F. Theoretical insight into the trends that guide the electrochemical reduction of carbon dioxide to formic acid. ChemSusChem 9, 358-363 (2016).

43. Cheng, T., Xiao, H. \& Goddard, W. A. Reaction mechanisms for the electrochemical reduction of $\mathrm{CO}_{2}$ to $\mathrm{CO}$ and formate on the $\mathrm{Cu}(100)$ surface at $298 \mathrm{~K}$ from quantum mechanics free energy calculations with explicit water. J. Am. Chem. Soc. 138, 13802-13805 (2016).

44. Bajdich, M., García-Mota, M., Vojvodic, A., Nørskov, J. K. \& Bell, A. T. Theoretical investigation of the activity of cobalt oxides for the electrochemical oxidation of water. J. Am. Chem. Soc. 135, 13521-13530 (2013).

45. Sumaria, V., Krishnamurthy, D. \& Viswanathan, V. Quantifying confidence in DFT predicted surface Pourbaix diagrams and associated reaction pathways for Chlorine Evolution. ACS Catal. 8, 9034-9042 (2018).

46. Persson, K. A., Waldwick, B., Lazic, P. \& Ceder, G. Prediction of solid-aqueous equilibria: Scheme to combine first-principles calculations of solids with experimental aqueous states. Phys. Rev. B - Condens. Matter Mater. Phys. 85, 1-12 (2012)

47. Singh, A. K. et al. Electrochemical stability of metastable materials. Chem. Mater. 29, 10159-10167 (2017).

48. Kresse, G. \& Hafner, J. Ab initio molecular dynamics for liquid metals. Phys. Rev. B 47, 558 (1993).

49. Blöchl, P. E. Projector augmented-wave method. Phys. Rev. B 50, 17953-17979 (1994).

50. Perdew, J. P., Burke, K. \& Ernzerhof, M. Generalized gradient approximation made simple. Phys. Rev. Lett. 77, 3865-3868 (1996).

51. Togo, A., Oba, F. \& Tanaka, I. First-principles calculations of the ferroelastic transition between rutile-type and $\mathrm{CaCl}_{2}$-type $\mathrm{SiO}_{2}$ at high pressures. Phys Rev. B 78, 134106 (2008).

52. Martyna, G. J., Klein, M. L. \& Tuckerman, M. Nosé-Hoover chains: the canonical ensemble via continuous dynamics. J. Chem. Phys. 97, 2635-2643 (1992).

53. Mathew, K., Sundararaman, R., Letchworth-Weaver, K., Arias, T. A. \& Hennig, R. G. Implicit solvation model for density-functional study of nanocrystal surfaces and reaction pathways. J. Chem. Phys. 140, 084106 (2014).

54. Henkelman, G., Uberuaga, B. P. \& Jónsson, H. Climbing image nudged elastic band method for finding saddle points and minimum energy paths. J. Chem. Phys. 113, 9901-9904 (2000).

55. Sundararaman, R. et al. JDFTx: Software for joint density-functional theory. SoftwareX 6, 278-284 (2017).

56. Sundararaman, R. \& Schwarz, K. Evaluating continuum solvation models for the electrode-electrolyte interface: challenges and strategies for improvement. J. Chem. Phys. 146, 08411 (2017).

57. Nørskov, J. K. et al. Origin of the overpotential for oxygen reduction at a fuelcell cathode. J. Phys. Chem. B 108, 17886-17892 (2004). 
58. Valdés, Á., Qu, Z.-W., Kroes, G.-J., Rossmeisl, J. \& Nørskov, J. K. Oxidation and photo-oxidation of water on $\mathrm{TiO}_{2}$ surface. J. Phys. Chem. C 112, 9872-9879 (2008).

\section{Acknowledgements}

We are grateful for funding support from the National Key R\&D Program of China (2019YFA0308000), the Natural Science Foundation of China (No. 21873050), and the Priority Academic Program Development of Jiangsu Higher Education Institutions.

\section{Author contributions}

Y.L. designed the research, X.R.Z., X.C.Z., and Y.J. demonstrated the initial idea and collected all the data. X.R.Z. and Y.L. wrote the paper and all authors commented on it.

\section{Competing interests}

The authors declare no competing interests.

\section{Additional information}

Supplementary information The online version contains supplementary material available at https://doi.org/10.1038/s41467-021-24400-5.

Correspondence and requests for materials should be addressed to Y.L.
Peer review information Nature Communications thanks Joseph Montoya and the other anonymous reviewers for their contribution to the peer review of this work.

Reprints and permission information is available at http://www.nature.com/reprints

Publisher's note Springer Nature remains neutral with regard to jurisdictional claims in published maps and institutional affiliations.

(c) (i) Open Access This article is licensed under a Creative Commons Attribution 4.0 International License, which permits use, sharing, adaptation, distribution and reproduction in any medium or format, as long as you give appropriate credit to the original author(s) and the source, provide a link to the Creative Commons license, and indicate if changes were made. The images or other third party material in this article are included in the article's Creative Commons license, unless indicated otherwise in a credit line to the material. If material is not included in the article's Creative Commons license and your intended use is not permitted by statutory regulation or exceeds the permitted use, you will need to obtain permission directly from the copyright holder. To view a copy of this license, visit http://creativecommons.org/ licenses/by/4.0/.

(C) The Author(s) 2021 